\title{
CONCENTRATION PHENOMENA FOR FRACTIONAL ELLIPTIC EQUATIONS INVOLVING EXPONENTIAL CRITICAL GROWTH
}

\author{
CLAUDIANOR O. ALVES, JOÃO MARCOS DO Ó, AND OLÍMPIO H. MIYAGAKI
}

\begin{abstract}
In this paper, we deal with the following singular perturbed fractional elliptic problem $\varepsilon(-\Delta)^{1 / 2} u+V(z) u=f(u)$ in $\mathbb{R}$, where $(-\Delta)^{1 / 2} u$ is the square root of the Laplacian and $f(s)$ has exponential critical growth. Under suitable conditions on $f(s)$, we construct a localized bound state solution concentrating at an isolated component of the positive local minimum points of the potential of $V$ as $\varepsilon$ goes to 0 .
\end{abstract}

\section{Contents}

1. Introduction

1.1. Statement of the main the result

1.2. Outline

2. Preliminary results

3. The Caffarelli and Silvestre's method 6

4. Proof of Theorem $1.1 \quad 19$

References

\section{INTRODUCTION}

In this paper, we are concerned with existence and concentration of positive solutions for the following singular perturbed fractional elliptic problem

$$
\left\{\begin{aligned}
\varepsilon(-\Delta)^{1 / 2} u+V(z) u=f(u) & \text { in } \quad \mathbb{R}, \\
u \in H^{1 / 2}(\mathbb{R}), \quad u>0 & \text { on } \quad \mathbb{R},
\end{aligned}\right.
$$

where $\varepsilon$ is a small positive parameter, the potential $V$ is bounded away from zero, the nonlinearity $f(s)$ has exponential critical growth and $(-\Delta)^{1 / 2} u$ is the square root of the Laplacian, which may be defined for smooth functions as

$$
\mathcal{F}\left((-\Delta)^{1 / 2} u\right)(\xi)=|\xi| \mathcal{F}(u)(\xi)
$$

where $\mathcal{F}$ is the Fourier transform, that is,

$$
\mathcal{F}(\xi)=\frac{1}{\sqrt{2 \pi}} \int_{\mathbb{R}} \mathrm{e}^{-i \xi \cdot x} \phi(x) \mathrm{d} x,
$$

Date: September 11, 2018.

2000 Mathematics Subject Classification. 35J60, 35B09, 35B33, 35R11.

Key words and phrases. Trudinger-Moser inequality.

Research partially supported by INCTmat/MCT/Brazil, CNPq, CAPES/Brazil. 
for functions $\phi$ in the Schwartz class. Also, for sufficiently smooth $u,(-\Delta)^{1 / 2} u$ can be equivalently represented, see $[17,22]$, as

$$
(-\Delta)^{1 / 2} u=-\frac{1}{2 \pi} \int_{\mathbb{R}} \frac{u(x+y)+u(x-y)-2 u(x)}{|y|^{2}} \mathrm{~d} y,
$$

and, by [17, Propostion 3.6],

$$
\left\|(-\Delta)^{1 / 4} u\right\|_{L^{2}}^{2}:=\frac{1}{2 \pi} \int_{\mathbb{R}^{2}} \frac{(u(x)-u(y))^{2}}{|x-y|^{2}} \mathrm{~d} x \mathrm{~d} y, \quad \forall u \in H^{1 / 2}(\mathbb{R}) .
$$

Here $H^{1 / 2}(\mathbb{R})$ is the fractional Sobolev space

$$
H^{1 / 2}(\mathbb{R})=\left\{u \in L^{2}(\mathbb{R}):\left\|(-\Delta)^{1 / 4} u\right\|_{L^{2}}^{2}<\infty\right\},
$$

endowed with the norm

$$
\|u\|_{H^{1 / 2}}=\left(\|u\|_{L^{2}}^{2}+\left\|(-\Delta)^{1 / 4} u\right\|_{L^{2}}^{2}\right)^{1 / 2} .
$$

We suppose that the potential $V: \mathbb{R} \rightarrow \mathbb{R}$ is bounded and satisfies the following hypotheses:

$\left(V_{1}\right): V$ is locally Hölder continuous and there exists $V_{0}>0$ such that

$$
V(z) \geq V_{0}, \quad \forall z \in \mathbb{R},
$$

$\left(V_{2}\right)$ : there exists a bounded interval $\Lambda \subset \mathbb{R}$ such that

$$
V_{0} \equiv \inf _{\Lambda} V(z)<\min _{\partial \Lambda} V(z) \text {. }
$$

The function $f: \mathbb{R} \rightarrow \mathbb{R}$ satisfies the so-called Ambrosetti-Rabinowitz condition, introduced in [5], namely,

$$
\text { there exists } \vartheta>2 \text { with } 0<\vartheta F(s) \leq s f(s) \text { for all } s>0, \quad F(s)=\int_{0}^{s} f(t) \mathrm{d} t .
$$

In addition to the above condition we make the following assumptions on $f$ :

(f1) : $f: \mathbb{R} \rightarrow \mathbb{R}^{+}$is $C^{1}$ function with $f(s)=0$ if $s<0$.

(f2) : $f(s)=o(s)$ near origin.

(f3): $f(s) / s$ is an increasing function in $\mathbb{R}^{+}$.

(f4): There exist constants $p>2$ and $C_{p}>0$ such that

$$
f(s) \geq C_{p} s^{p-1} \text { for all } s>0,
$$

where

$$
C_{p}>\left[\beta_{p}\left(\frac{2 \vartheta}{\vartheta-2}\right) \frac{1}{\min \left\{1, V_{0}\right\}}\right]^{(p-2) / 2},
$$

with

$$
\begin{gathered}
\beta_{p}=\inf _{\mathcal{N}_{0}} \tilde{J}_{0} \\
\mathcal{N}_{0}=\left\{v \in X^{1}\left(\mathbb{R}_{+}^{2}\right) \backslash\{0\}: \tilde{J}_{0}^{\prime}(v) v=0\right\}
\end{gathered}
$$

and

$$
\tilde{J}_{0}(v)=\frac{1}{2} \int_{\mathbb{R}_{+}^{2}}|\nabla v|^{2} \mathrm{~d} x \mathrm{~d} y+\frac{1}{2} \int_{\mathbb{R}} V_{0}|v(x, 0)|^{2} \mathrm{~d} x-\frac{1}{p} \int_{\mathbb{R}}|v(x, 0)|^{p} \mathrm{~d} x .
$$

where $X^{1}\left(\mathbb{R}_{+}^{2}\right)$ is defined in $(2.1)$. 
We are interested in bound state solution of $\left(P_{\varepsilon}\right)$ (solution with finite energy), when $f$ has the maximal growth which allow us to treat problem $\left(P_{\varepsilon}\right)$ variationally in the fractional Sobolev space $H^{1 / 2}(\mathbb{R})$ motivated by the following Trudinger-Moser type inequality due to T. Ozawa [24].

Theorem A. There exists $0<\omega \leq \pi$ such that, for all $\alpha \in(0, \omega)$, there exists $H_{\alpha}>0$ with

$$
\int_{\mathbb{R}}\left(\mathrm{e}^{\alpha u^{2}}-1\right) \mathrm{d} x \leq H_{\alpha}\|u\|_{L^{2}}^{2},
$$

for all $u \in H^{1 / 2}(\mathbb{R})$ with $\left\|(-\Delta)^{1 / 4} u\right\|_{L^{2}}^{2} \leq 1$.

In view of (1.1), we say that $f$ has exponential critical growth at $+\infty$, if there exist $\omega \in(0, \pi)$ and $\alpha_{0} \in(0, \omega)$, such that

$$
\lim _{s \rightarrow+\infty} \frac{f(s)}{\mathrm{e}^{\alpha s^{2}}}=0, \forall \alpha>\alpha_{0}, \quad \text { and } \quad \lim _{s \rightarrow+\infty} \frac{f(s)}{\mathrm{e}^{\alpha s^{2}}}=+\infty, \forall \alpha<\alpha_{0} .
$$

1.1. Statement of the main the result. The following theorem contains our main result:

Theorem 1.1. Assume $\left(V_{1}\right),\left(V_{2}\right),(A R)$, and $(f 1)-(f 4)$ hold. Then there exists $\varepsilon_{0}>0$ such that for $\varepsilon \in\left(0, \varepsilon_{0}\right)$, problem $\left(P_{\varepsilon}\right)$ possesses a positive bound state solution $u_{\varepsilon}(z)$ verifying the following conditions

I): $u_{\varepsilon}$ has at most one local (hence global) maximum $z_{\varepsilon}$ in $\mathbb{R}$ and $z_{\varepsilon} \in I$,

II): $\lim _{\varepsilon \rightarrow 0^{+}} V\left(z_{\varepsilon}\right)=V_{1}=\inf _{I} V$,

This result extends to the nonlocal case the main result in [19]. The proof is made combining Ozawa inequality [24] with Del Pino and Felmer [16] truncation argument and a recent approach developed in Alves and Miyagaki [4]. In [12,25,27] were established existence results in nonlocal situation, while in $[13,14,20,26]$ a concentration phenomena were proved imposing a global condition in $V$.

Remark 1.2. (1) We recall that the condition (AR) impose some superquadratic growth condition on the nonlinearity $F$.

(2) The condition ( $f 4)$ appeared first in [11], then for instance in [2] and [19]. For the nonlocal situation it was used, e.g., in [18].

(3) Critical growth of Trudinger-Moser type was used in [15], also in [1, 2, 19]. In [23] and in [18] were used the Ozawa inequality to discuss nonlocal problem in bounded and unbounded domain, respectively.

(4) Notice that, if $f(s)$ has exponential critical growth, instead of assumption ( $f 4)$, it is enough to assume that there exist $p>2$ and $\mu>0$ such that

$$
\liminf _{s \rightarrow 0^{+}} \frac{f(s)}{s^{p-1}} \geq \mu \text {. }
$$

Throughout the paper, unless explicitly stated, the symbol $C$ will always denote a generic positive constant, which may vary from line to line.

1.2. Outline. The sequel of the paper is organized as follows. The next section contains some technical results, which are crucial tools to prove our main theorem. In Sect. 3, we adapt a method due to L. Caffarelli and L. Silvestre to obtain a local realization of the fractional Laplacian via a Dirichlet-to-Neummann operator. As a consequence of this argument we transform our nonlocal Problem $\left(P_{\varepsilon}\right)$ into one local problem defined on the upper half plane $\left(L P_{\varepsilon}\right)$. Using variational techniques combined with Del Pino and Felmer truncation argument we give the proof of Theorem 1.1 in Sect. 4, 


\section{Preliminary Results}

In this section we collect preliminary facts for future reference. First of all, let us set the standard notations to be used in the paper. We denote the upper half-space in $\mathbb{R}^{2}$ by $\mathbb{R}_{+}^{2}=\left\{(x, y) \in \mathbb{R}^{2}: y>0\right\}$. In the sequel, $X^{1}\left(\mathbb{R}_{+}^{2}\right)$ denotes the completion of $C_{0}^{\infty}\left(\overline{\mathbb{R}_{+}^{2}}\right)$ with relation to the norm $\|v\|_{\varepsilon}$,

$$
\begin{aligned}
X^{1}\left(\mathbb{R}_{+}^{2}\right) & :=\overline{C_{0}^{\infty}\left(\overline{\mathbb{R}_{+}^{2}}\right)}\left\|^{\|}\right\|_{\varepsilon}, \quad \text { where } \\
\|v\|_{\varepsilon} & :=\left(\int_{\mathbb{R}_{+}^{2}}|\nabla v(x, y)|^{2} \mathrm{~d} x \mathrm{~d} y+\int_{\mathbb{R}} V(\varepsilon x)|v(x, 0)|^{2} \mathrm{~d} x\right)^{1 / 2} .
\end{aligned}
$$

Moreover, we denote by \|\| the usual norm in $X^{1}\left(\mathbb{R}_{+}^{2}\right)$, that is,

$$
\|v\|=\left(\int_{\mathbb{R}_{+}^{2}}|\nabla v(x, y)|^{2} \mathrm{~d} x \mathrm{~d} y+\int_{\mathbb{R}}|v(x, 0)|^{2} \mathrm{~d} x\right)^{1 / 2} .
$$

Since the potential $V$ is bounded from above and below, it is easy to see that \|\|$_{\varepsilon}$ and \|\| are equivalent norms in $X^{1}\left(\mathbb{R}_{+}^{2}\right)$ with

$$
\min \left\{1, V_{0}\right\}\|v\| \leq\|v\|_{\varepsilon} \leq \min \left\{1,|V|_{\infty}\right\}\|v\|, \quad \forall v \in X^{1}\left(\mathbb{R}_{+}^{2}\right) .
$$

Using the above definition, we see that if $v \in X^{1}\left(\mathbb{R}_{+}^{2}\right)$, then $u(x)=v(x, 0)$ belongs to $H^{1 / 2}(\mathbb{R})$ and

$$
\|v\|=\|u\|_{H^{1 / 2}} .
$$

Since $H^{1 / 2}(\mathbb{R})$ is continuously embedded into $L^{q}(\mathbb{R})$ for all $q \geq 2$, c.f. [17, Theorem 6.9], it follows that $X^{1}\left(\mathbb{R}_{+}^{2}\right)$ is also continuously embedded into $L^{q}(\mathbb{R})$ for all $q \geq 2$. Moreover, the embedded

$$
X^{1}\left(\mathbb{R}_{+}^{2}\right) \hookrightarrow L^{q}(A)
$$

are compact for any bounded mensurable set $A \subset \mathbb{R}$. See [22, Proposition 3.6] also [18, Remark 2.1].

Our first lemma is an important Trudinger-Moser inequality on $X^{1}\left(\mathbb{R}_{+}^{2}\right)$, which was proved in [18, Lemma 2.4].

Lemma 2.1. Let $\left(v_{n}\right) \subset X^{1}\left(\mathbb{R}_{+}^{2}\right)$ be a bounded sequence and assume $\sup _{n \in \mathbb{N}}\left\|v_{n}\right\|^{2}=M$. Then

$$
\sup _{n \in \mathbb{N}} \int_{\mathbb{R}}\left(e^{\alpha\left|v_{n}(x, 0)\right|^{2}}-1\right) \mathrm{d} x<\infty, \quad \text { for every } 0<\alpha<\frac{\omega}{M^{2}}
$$

In particular, if $M \in(0,1)$, there exists $\alpha_{M}<\omega$ such that

$$
\sup _{n \in \mathbb{N}} \int_{\mathbb{R}}\left(e^{\alpha_{M}\left|v_{n}(x, 0)\right|^{2}}-1\right) \mathrm{d} x<\infty .
$$

Using the above lemma, we are able to prove some technical lemmas. The first of them is crucial in the study the behavior of Palais-Smale sequences.

Lemma 2.2. Let $\left(v_{n}\right)$ be a sequence in $X^{1}\left(\mathbb{R}_{+}^{2}\right)$ with

$$
\limsup _{n \rightarrow+\infty}\left\|v_{n}\right\|^{2}<1
$$

Then, there exist $t>1$ sufficiently close to 1 and $C>0$ satisfying

$$
\int_{\mathbb{R}}\left(e^{\omega\left|v_{n}(x, 0)\right|^{2}}-1\right)^{t} \mathrm{~d} x \leq C, \quad \forall n \in \mathbb{N} .
$$


Proof. Using (2.3) there are $m>0$ and $n_{0} \in \mathbb{N}$ verifying

$$
\left\|v_{n}\right\|^{2}<m<1, \quad \forall n \geq n_{0} .
$$

Fix $t>1$ sufficiently close to 1 and $\beta>t$ satisfying $\beta m<1$. Then, there exists $C=C(\beta)>0$ such that

$$
\int_{\mathbb{R}}\left(e^{\omega\left|v_{n}(x, 0)\right|^{2}}-1\right)^{t} \mathrm{~d} x \leq C \int_{\mathbb{R}}\left(e^{\beta m \omega\left(\frac{\left|v_{n}(x, 0)\right|}{\left\|\mid v_{n}\right\|}\right)^{2}}-1\right) \mathrm{d} x,
$$

for every $n \geq n_{0}$. Hence, by Lemma 2.1,

$$
\int_{\mathbb{R}}\left(e^{\omega\left|v_{n}(x, 0)\right|^{2}}-1\right)^{t} \mathrm{~d} x \leq C_{1} \quad \forall n \geq n_{0},
$$

for some positive constant $C_{1}$. Now, the lemma follows fixing

$$
C=\max \left\{C_{1}, \int_{\mathbb{R}}\left(e^{\omega\left|v_{1}\right|^{2}}-1\right)^{t} \mathrm{~d} x, \ldots, \int_{\mathbb{R}}\left(e^{\omega\left|v_{n_{0}}\right|^{2}}-1\right)^{t} \mathrm{~d} x\right\} .
$$

Corollary 2.3. Let $\left(v_{n}\right)$ be a sequence in $X^{1}\left(\mathbb{R}_{+}^{2}\right)$ satisfying $(2.3)$. If $v_{n} \rightarrow v$ weakly in $X^{1}\left(\mathbb{R}_{+}^{2}\right)$ and $v_{n}(x, 0) \rightarrow v(x, 0)$ a.e in $\mathbb{R}$, as $n \rightarrow \infty$, then,

$$
\begin{aligned}
F\left(v_{n}(x, 0)\right) & \rightarrow F(v(x, 0)) \quad \text { in } \quad L^{1}(-R, R), \\
f\left(v_{n}(x, 0)\right) v_{n}(x, 0) & \rightarrow f(v(x, 0)) v(x, 0) \quad \text { in } \quad L^{1}(-R, R)
\end{aligned}
$$

and

$$
\int_{-R}^{R} f\left(v_{n}(x, 0)\right) \phi(x, 0) \rightarrow \int_{-R}^{R} f(v(x, 0)) \phi(x, 0),
$$

as $n \rightarrow \infty$, for all $\phi \in X^{1}\left(\mathbb{R}_{+}^{2}\right)$ and $R>0$.

Proof. By $\left(f_{1}\right)$, for each $\beta>1$ and $\alpha>\alpha_{0}$, there is $C>0$ such that

$$
|F(s)| \leq C\left(|s|^{2}+\left(e^{\alpha \beta|s|^{2}}-1\right)\right) \quad \forall s \in \mathbb{R}
$$

from where it follows that,

$$
\left|F\left(v_{n}(x, 0)\right)\right| \leq C\left(\left|v_{n}(x, 0)\right|^{2}+\left(e^{\alpha \beta\left|v_{n}(x, 0)\right|^{2}}-1\right)\right), \quad \forall n \in \mathbb{N} .
$$

Setting

$$
h_{n}(x)=C\left(e^{\alpha_{0} \beta\left|v_{n}(x, 0)\right|^{2}}-1\right),
$$

we can fix $\beta, q>1$ sufficiently close to 1 and $\alpha$ sufficiently close to $\alpha_{0}$ such that

$$
h_{n} \in L^{q}(\mathbb{R}) \quad \text { and } \sup _{n \in \mathbb{N}}\left|h_{n}\right|_{q}<+\infty,
$$

which is an immediate consequence of Lemma 2.2. Therefore, up to subsequence, we derive that

$$
h_{n} \rightarrow h=C\left(e^{\alpha_{0} \beta|v(x, 0)|^{2}}-1\right) \quad \text { weakly in } \quad L^{q}(\mathbb{R}), \text { as } n \rightarrow \infty .
$$

Since $h_{n}, h \geq 0$, the last limit yields

$$
h_{n} \rightarrow h \quad \text { in } L^{1}(-R, R), \quad \forall R>0, \text { as } n \rightarrow \infty .
$$

On the other hand, we know that

$$
v_{n}(\cdot, 0) \rightarrow v(\cdot, 0) \quad \text { in } \quad L^{2}(-R, R), \text { as } n \rightarrow \infty .
$$

Gathering the above limits with (2.7), we get

$$
F\left(v_{n}(x, 0)\right) \rightarrow F(v(x, 0)) \quad \text { in } \quad L^{1}(-R, R), \quad \forall R>0, \text { as } n \rightarrow \infty .
$$


The limits (2.5) and (2.6) follow with the same type of arguments.

The next lemma is a Lions type result, which is crucial in our approach. Since it follows with the same arguments found in C. Alves, J. M. do Ó and O. Miyagaki [2, Proposition 2.3], we will omit its proof.

Lemma 2.4. Let $\left(v_{n}\right) \subset X^{1}\left(\mathbb{R}_{+}^{2}\right)$ be a sequence with

$$
\limsup _{n \rightarrow+\infty}\left\|v_{n}\right\|^{2}<1
$$

If there is $R>0$ such that

$$
\lim _{n \rightarrow+\infty} \sup _{z \in \mathbb{R}} \int_{z-R}^{z+R}\left|v_{n}(x, 0)\right|^{2} \mathrm{~d} x=0
$$

then

$$
\lim _{n \rightarrow+\infty} \int_{\mathbb{R}} F\left(v_{n}(x, 0)\right) \mathrm{d} x=\lim _{n \rightarrow+\infty} \int_{\mathbb{R}} f\left(v_{n}(x, 0)\right) v_{n}(x, 0) \mathrm{d} x=0 .
$$

\section{The Caffarelli and Silvestre's method}

First of all, using the change variable $u(x)=v(\varepsilon x)$, it is possible to prove that Problem $\left(P_{\varepsilon}\right)$ is equivalent to the problem

$$
\left\{\begin{array}{rll}
(-\Delta)^{1 / 2} u+V(\varepsilon z) u=f(u) & \text { in } & \mathbb{R}, \\
u \in H^{1 / 2}(\mathbb{R}), \quad u>0 & \text { on } & \mathbb{R} .
\end{array}\right.
$$

Hereafter, to get a solution for $\left(P_{\varepsilon}^{\prime}\right)$, we will use a method due to L. Caffarelli and L. Silvestre in [10], more exactly, due to R. Frank and E. Lenzmann [22] for whole line. In the seminal above papers, were developed a local interpretation of the fractional Laplacian given in $\mathbb{R}$ by considering a Dirichlet to Neumann type operator in the domain $\mathbb{R}_{+}^{2}=\left\{(x, t) \in \mathbb{R}^{2}: t>0\right\}$. A similar extension, in a bounded domain, see for instance, $[6,8,9]$. For $u \in H^{1 / 2}(\mathbb{R})$, the solution $w \in X^{1}\left(\mathbb{R}_{+}^{2}\right)$ of

$$
\left\{\begin{array}{rll}
-\operatorname{div}(\nabla w)=0 & \text { in } & \mathbb{R}_{+}^{2} \\
w=u & \text { on } & \mathbb{R} \times\{0\}
\end{array}\right.
$$

is called $1 / 2$-harmonic extension $w=E_{1 / 2}(u)$ of $u$ and it is proved in [10] that

$$
\lim _{y \rightarrow 0^{+}} \frac{\partial w}{\partial y}(x, y)=-(-\Delta)^{1 / 2} u(x) .
$$

To get a solution for the nonlocal Problem $\left(P_{\varepsilon}^{\prime}\right)$, we will study the existence of solution for the local problem defined on the upper half plane

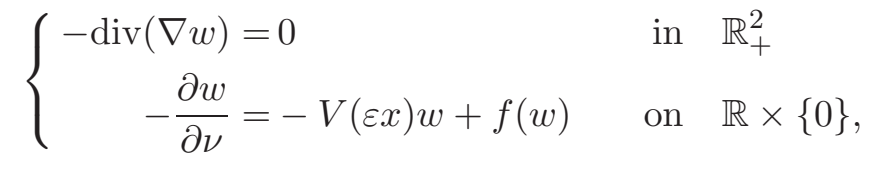

where

$$
\frac{\partial w}{\partial \nu}=\lim _{y \rightarrow 0^{+}} \frac{\partial w}{\partial y}(x, y)
$$

since if $w$ is a solution for the above problem, the function $u(x)=w(x, 0)$ is a solution for $\left(P_{\varepsilon}^{\prime}\right)$. 
Associated with $\left(L P_{\varepsilon}\right)$, we have the $J_{\varepsilon}: X^{1}\left(\mathbb{R}_{+}^{2}\right) \rightarrow \mathbb{R}$ defined by

$$
J_{\varepsilon}(v)=\frac{1}{2} \int_{\mathbb{R}_{+}^{2}}|\nabla v|^{2} \mathrm{~d} x \mathrm{~d} y+\frac{1}{2} \int_{\mathbb{R}} V(\varepsilon x)|v(x, 0)|^{2} \mathrm{~d} x-\int_{\mathbb{R}} F(v(x, 0)) \mathrm{d} x,
$$

which is $C^{1}\left(X^{1}\left(\mathbb{R}_{+}^{2}\right), \mathbb{R}\right)$ with derivative given by

$$
\begin{aligned}
J_{\varepsilon}^{\prime}(v) \phi= & \frac{1}{2} \int_{\mathbb{R}_{+}^{2}} \nabla v \cdot \nabla \phi \mathrm{d} x \mathrm{~d} y \\
& +\frac{1}{2} \int_{\mathbb{R}} V(\varepsilon x) v(x, 0) \phi(x, 0) \mathrm{d} x-\int_{\mathbb{R}} f(v(x, 0)) \phi(x, 0) \mathrm{d} x, \quad \forall \phi \in X^{1}\left(\mathbb{R}_{+}^{2}\right) .
\end{aligned}
$$

We would like point out that $u$ is a solution of $\left(P_{\varepsilon}^{\prime}\right)$ if, and only if, $u=v(x, 0)$ for all $x \in \mathbb{R}$, for some critical point $v$ of $J_{\varepsilon}$.

In what follows, we will not work directly with functional $J_{\varepsilon}$, because we have some difficulties to prove that it verifies the $(P S)$ condition. Hereafter, we will use the same approach explored in [16], modifying the nonlinearity of a suitable way. The idea is the following:

First of all, without loss of generality, we will assume that

$$
0 \in \Lambda \quad \text { and } \quad V(0)=V_{0}=\inf _{x \in \mathbb{R}} V(x) .
$$

We recall that in assumption $(f 1)$ we imposed that $f(t)=0, \forall t \leq 0$, because we are looking for positive solutions. Moreover, let us choose $k>2 \vartheta /(\vartheta-2)$ and $a>0$ verifying

$$
\frac{f(a)}{a}=\frac{V_{0}}{k}
$$

where $V_{0}>0$ was given in $\left(V_{1}\right)$. Using these numbers, we set the functions

$$
\tilde{f}(t)=\left\{\begin{array}{l}
f(t), \quad t \leq a, \\
\frac{V_{0}}{k} t, \quad t \geq a
\end{array}\right.
$$

and

$$
g(x, t)=\chi_{\Lambda}(x) f(t)+\left(1-\chi_{\Lambda}\right) \tilde{f}(t), \quad \forall(x, t) \in \mathbb{R}^{2},
$$

where $\Lambda$ was given in $\left(V_{2}\right)$ and $\chi_{\Lambda}$ denotes the characteristic function associated with $\Lambda$, that is,

$$
\chi(x)= \begin{cases}1, & x \in \Lambda \\ 0, & x \in \Lambda^{c}\end{cases}
$$

Using the above functions, we will study the existence of positive solution for the following problem

$$
\left\{\begin{array}{l}
(-\Delta)^{1 / 2} u+V(\varepsilon x) u=g_{\varepsilon}(x, u), \quad x \in \mathbb{R}, \\
u \in H^{1 / 2}(\mathbb{R}),
\end{array}\right.
$$

where

$$
g_{\varepsilon}(x, t)=g(\varepsilon x, t), \quad \forall(x, t) \in \mathbb{R}^{2} .
$$

We recall by using [10], to get a solution for the above problem, it is enough to study the existence of solution for the problem

$$
\left\{\begin{array}{rll}
-\operatorname{div}(\nabla w)=0 & \text { in } & \mathbb{R}_{+}^{2} \\
\frac{\partial w}{\partial \nu}=V(\varepsilon x) w-g_{\varepsilon}(x, w) & \text { on } & \mathbb{R} \times\{0\}
\end{array}\right.
$$

because if $w$ is a solution of $(A P)^{\prime}$, the function $u(x)=w(x, 0)$ is a solution for $(A P)$. 
Here, we would like point out that if $v_{\varepsilon} \in X^{1}\left(\mathbb{R}_{+}^{2}\right)$ is a solution of $(A P)^{\prime}$ with

$$
v_{\varepsilon}(x, 0)<a, \quad \forall x \in \Lambda_{\varepsilon}^{c},
$$

where $\Lambda_{\varepsilon}=\Lambda / \varepsilon$, then $u_{\varepsilon}(x)=v_{\varepsilon}(x, 0)$ is a solution of $\left(P_{\varepsilon}^{\prime}\right)$.

Associated with $(A P)^{\prime}$, we have the energy functional $E_{\varepsilon}: X^{1}\left(\mathbb{R}_{+}^{2}\right) \rightarrow \mathbb{R}$ given by

$$
E_{\varepsilon}(v)=\frac{1}{2} \int_{\mathbb{R}_{+}^{2}}|\nabla v|^{2} \mathrm{~d} x \mathrm{~d} y+\frac{1}{2} \int_{\mathbb{R}} V(\varepsilon x)|v(x, 0)|^{2} \mathrm{~d} x-\int_{\mathbb{R}} G_{\varepsilon}(x, v(x, 0)) \mathrm{d} x
$$

where

$$
G_{\varepsilon}(x, t)=\int_{0}^{t} g_{\varepsilon}(x, \tau) \mathrm{d} \tau, \quad \forall(x, t) \in \mathbb{R}^{2} .
$$

Using the definition of $g$, it follows that

$\left(g_{1}\right) \quad \vartheta G_{\varepsilon}(x, t) \leq g_{\varepsilon}(x, t) t, \quad \forall(x, t) \in \Lambda_{\varepsilon} \times \mathbb{R}$,

$\left(g_{2}\right) \quad 2 G_{\varepsilon}(x, t) \leq g_{\varepsilon}(x, t) t \leq \frac{V_{0}}{k}|t|^{2}, \quad \forall(x, t) \in\left(\Lambda_{\varepsilon}\right)^{c} \times \mathbb{R}$.

From assumption $\left(g_{2}\right)$,

$$
\begin{aligned}
& L(x, t)=V(x)-G_{\varepsilon}(x, t) \geq\left(1-\frac{1}{2 k}\right) V(x)|t|^{2} \geq 0, \quad \forall(x, t) \in\left(\Lambda_{\varepsilon}\right)^{c} \times \mathbb{R}, \\
& M(x, t)=V(x)-g_{\varepsilon}(x, t) t \geq\left(1-\frac{1}{k}\right) V(x)|t|^{2} \geq 0, \quad \forall(x, t) \in\left(\Lambda_{\varepsilon}\right)^{c} \times \mathbb{R} .
\end{aligned}
$$

Lemma 3.1. The functional $E_{\varepsilon}$ verifies the mountain pass geometry, that is,

i) There are $r, \rho>0$ such that

$$
E_{\varepsilon}(v) \geq \rho, \quad \text { for } \quad\|v\|=r
$$

ii) There is $e \in X^{1}\left(\mathbb{R}_{+}^{2}\right)$ with $\|e\|>r$ and $E_{\varepsilon}(e)<0$.

Proof. From $\left(g_{1}\right)-\left(g_{4}\right)$, there exist $c_{1}, c_{2}>0$ verifying

$$
E_{\varepsilon}(v) \geq c_{1}\|v\|^{2}-c_{2}\|v\|^{q}, \quad \forall v \in X^{1}\left(\mathbb{R}_{+}^{2}\right) .
$$

From the above inequality, there are $r, \rho>0$ such that

$$
E_{\varepsilon}(v) \geq \rho, \quad \text { for } \quad\|v\|_{1, s}=r,
$$

showing $i$ ). To prove $i i)$, fix $\varphi \in X^{1}\left(\mathbb{R}_{+}^{2}\right)$ with supp $\varphi \subset \Lambda_{\varepsilon} \times \mathbb{R}$. Then, for $t>0$

$$
E_{\varepsilon}(t \varphi)=\frac{t^{2}}{2}\|\varphi\|^{2}-\int_{\mathbb{R}} F(t \varphi(x, 0)) \mathrm{d} x .
$$

From $\left(f_{3}\right)$, we know that there are $c_{3}, c_{4} \geq 0$ verifying

$$
F(t) \geq c_{1}|t|^{\vartheta}-c_{2}, \quad \forall t \geq 0 .
$$

Using the above inequality, we derive

$$
\lim _{t \rightarrow+\infty} E_{\varepsilon}(t \varphi)=-\infty .
$$

Thereby, ii) follows with $e=t \varphi$ and $t$ large enough. 
In what follows, we denote by $c_{\varepsilon}$ the mountain pass level associated with $E_{\varepsilon}$. Related to the case $\varepsilon=0$, it is possible to prove that there is $w_{0} \in X^{1}\left(\mathbb{R}_{+}^{2}\right)$ such that

$$
J_{0}\left(w_{0}\right)=c_{0} \quad \text { and } \quad J_{0}^{\prime}\left(w_{0}\right)=0 .
$$

The existence of $w_{0}$ can be obtained repeating the same approach explored in [2].

Lemma 3.2. The minimax level $c_{0}$ verifies

$$
0<c_{0}<\min \left\{1, V_{0}\right\}\left(\frac{1}{2}-\frac{1}{\vartheta}\right) .
$$

Proof. Consider $w_{*} \in X^{1}\left(\mathbb{R}_{+}^{2}\right)$ verifying

$$
\tilde{J}_{0}\left(w_{*}\right)=\beta_{p} \quad \text { and } \quad \tilde{J}_{0}^{\prime}\left(w_{*}\right)=0 .
$$

By characterization of $c_{0}$,

$$
c_{0} \leq \max _{t \geq 0} J_{0}\left(t w_{*}\right)
$$

Consequently, by $\left(f_{5}\right)$,

$$
c_{0} \leq \max _{t \geq 0}\left\{\frac{t^{2}}{2} \int_{\mathbb{R}_{+}^{2}}\left|\nabla w_{*}\right|^{2} \mathrm{~d} x \mathrm{~d} y+\frac{1}{2} \int_{\mathbb{R}} V_{0}\left|w_{*}(x, 0)\right|^{2} \mathrm{~d} x-\frac{C_{p} t^{p}}{p} \int_{\mathbb{R}}\left|w_{*}(x, 0)\right|^{p} \mathrm{~d} x\right\},
$$

which implies that

$$
c_{0} \leq C_{p}^{2 /(2-p)} \beta_{p}
$$

Hence, from $\left(f_{5}\right)$,

$$
0<c_{0}<\min \left\{1, V_{0}\right\}\left(\frac{1}{2}-\frac{1}{\vartheta}\right)
$$

Hereafter, we will assume that $k$ is large enough such that

$$
0<c_{0}<\min \left\{1, V_{0}\right\}\left(\left(\frac{1}{2}-\frac{1}{\vartheta}\right)-\frac{1}{k}\right)<\min \left\{1, V_{0}\right\}\left(\frac{1}{2}-\frac{1}{\vartheta}\right) .
$$

The next lemma establishes an important relation between $c_{\varepsilon}$ and $c_{0}$.

Lemma 3.3. The numbers $c_{0}$ and $c_{\varepsilon}$ verify the equality below

$$
\lim _{\varepsilon \rightarrow 0} c_{\varepsilon}=c_{0} .
$$

Hence, there is $\varepsilon_{0}>0$ such that

$$
0<\sup _{\varepsilon \in\left(0, \varepsilon_{0}\right)} c_{\varepsilon}<\min \left\{1, V_{0}\right\}\left(\left(\frac{1}{2}-\frac{1}{\vartheta}\right)-\frac{1}{k}\right) .
$$

Proof. From $\left(V_{1}\right)$,

$$
c_{\varepsilon} \geq c_{0}, \quad \forall \varepsilon \geq 0 .
$$

Then,

$$
\liminf _{\varepsilon \rightarrow 0} c_{\varepsilon} \geq c_{0}
$$

Next, fix $t_{\varepsilon}>0$ such that

$$
t_{\varepsilon} w \in \mathcal{M}_{\varepsilon}=\left\{v \in X^{1}\left(\mathbb{R}_{+}^{2}\right) \backslash\{0\}: E_{\varepsilon}^{\prime}(v) v=0\right\} .
$$


By definition of $c_{\varepsilon}$, we know that

$$
c_{\varepsilon} \leq \max _{t \geq 0} E_{\varepsilon}(t w)=E_{\varepsilon}\left(t_{\varepsilon} w\right) .
$$

Now standard arguments as those used in [19], it is possible to prove that

$$
\lim _{\varepsilon \rightarrow 0} t_{\varepsilon}=1
$$

and

$$
\lim _{\varepsilon \rightarrow 0} E_{\varepsilon}\left(t_{\varepsilon} w\right)=J_{0}(w)
$$

Thus,

$$
\limsup _{\varepsilon \rightarrow 0} c_{\varepsilon} \leq J_{0}(w)=c_{0}
$$

From (3.8) and (3.9),

$$
\limsup _{\varepsilon \rightarrow 0} c_{\varepsilon}=c_{0}
$$

showing (3.6). The inequality (3.7) is an immediate consequence of (3.6) and Lemma 3.2.

Lemma 3.4. Let $\varepsilon \in\left(0, \varepsilon_{0}\right)$ and $\left(v_{n}\right) \subset X^{1}\left(\mathbb{R}_{+}^{2}\right)$ be a $(P S)_{c_{\varepsilon}}$ sequence for $E_{\varepsilon}$. Then,

$$
\limsup _{n \rightarrow+\infty}\left\|v_{n}\right\|^{2}<1 \text {. }
$$

Proof. Gathering $E_{\varepsilon}\left(u_{n}\right)-\frac{1}{\vartheta} E_{\varepsilon}^{\prime}\left(u_{n}\right) u_{n}=c_{\varepsilon}+o_{n}(1)$ with definition of $g$, we find

$$
\left(\frac{1}{2}-\frac{1}{\vartheta}\right) \int_{\mathbb{R}_{+}^{2}}\left|\nabla v_{n}\right|^{2} \mathrm{~d} x \mathrm{~d} y+\left(\left(\frac{1}{2}-\frac{1}{\vartheta}\right)-\frac{1}{k}\right) V_{0} \int_{\mathbb{R}}\left|v_{n}(x, 0)\right|^{2} \mathrm{~d} x \leq c_{\varepsilon}+o_{n}(1),
$$

from where it follows that

$$
\min \left\{1, V_{0}\right\}\left(\left(\frac{1}{2}-\frac{1}{\vartheta}\right)-\frac{1}{k}\right) \limsup _{n \rightarrow+\infty}\left\|v_{n}\right\|^{2} \leq c_{\varepsilon}<\min \left\{1, V_{0}\right\}\left(\left(\frac{1}{2}-\frac{1}{\vartheta}\right)-\frac{1}{k}\right),
$$

and so,

$$
\limsup _{n \rightarrow+\infty}\left\|v_{n}\right\|^{2}<1
$$

Lemma 3.5. For $\varepsilon \in\left(0, \varepsilon_{0}\right)$, the functional $E_{\varepsilon}$ verifies the $(P S)_{c_{\varepsilon}}$ condition.

Proof. Let $\left(v_{n}\right) \subset X^{1}\left(\mathbb{R}_{+}^{2}\right)$ be a $(P S)_{c_{\varepsilon}}$ sequence for $E_{\varepsilon}$, that is,

$$
E_{\varepsilon}\left(v_{n}\right) \rightarrow c_{\varepsilon} \text { and } \quad E_{\varepsilon}^{\prime}\left(v_{n}\right) \rightarrow 0, \text { as } n \rightarrow \infty .
$$

From Lemma $3.4,\left(v_{n}\right)$ is bounded in $X^{1}\left(\mathbb{R}_{+}^{2}\right)$ and

$$
\limsup _{n \rightarrow+\infty}\left\|v_{n}\right\|^{2}<1
$$

Since $X^{1}\left(\mathbb{R}_{+}^{2}\right)$ is reflexive, there is a subsequence of $\left(v_{n}\right)$, still denoted by itself, and $v \in X^{1}\left(\mathbb{R}_{+}^{2}\right)$ such that

$$
\begin{aligned}
v_{n} \rightarrow v & \text { weakly in } \quad X^{1}\left(\mathbb{R}_{+}^{2}\right), \text { as } n \rightarrow \infty, \\
v_{n} \rightarrow v \quad \text { in } & L_{l o c}^{q}(\mathbb{R}), \quad \forall q \in[2,+\infty), \text { as } n \rightarrow \infty,
\end{aligned}
$$

and

$$
v_{n}(x, 0) \rightarrow v(x, 0) \quad \text { a.e. in } \mathbb{R} \text {, as } n \rightarrow \infty .
$$

Moreover, by Lemma 2.3,

$$
\int_{\mathbb{R}} f\left(v_{n}(x, 0)\right) \phi(x, 0) \mathrm{d} x \rightarrow \int_{\mathbb{R}} f(v(x, 0)) \phi(x, 0) \mathrm{d} x,
$$


as $n \rightarrow \infty$, for all $\phi \in C_{0}^{\infty}\left(\overline{\mathbb{R}_{+}^{2}}\right)$.

Using the above limits, it is possible to prove that $v$ is a critical point for $E_{\varepsilon}$, that is,

$$
E_{\varepsilon}^{\prime}(v) \varphi=0, \quad \forall \varphi \in X^{1}\left(\mathbb{R}_{+}^{2}\right) .
$$

Considering $\varphi=v$, we have that $E_{\varepsilon}^{\prime}(v) v=0$, and so,

$$
\begin{aligned}
& \int_{\mathbb{R}_{+}^{2}}|\nabla v|^{2} \mathrm{~d} x \mathrm{~d} y+\int_{\Lambda_{\varepsilon}} V(\varepsilon x)|v(x, 0)|^{2} \mathrm{~d} x+\int_{\left(\Lambda_{\varepsilon}\right)^{c}} M(x, v(x, 0)) \mathrm{d} x \\
& =\int_{\Lambda_{\varepsilon}} f(v(x, 0)) v(x, 0) \mathrm{d} x .
\end{aligned}
$$

On the other hand, using the limit $E_{\varepsilon}^{\prime}\left(v_{n}\right) v_{n}=o_{n}(1)$, we derive that

$$
\begin{aligned}
& \int_{\mathbb{R}_{+}^{2}}\left|\nabla v_{n}\right|^{2} \mathrm{~d} x \mathrm{~d} y+\int_{\Lambda_{\varepsilon}} V(\varepsilon x)\left|v_{n}(x, 0)\right|^{2} \mathrm{~d} x+\int_{\left(\Lambda_{\varepsilon}\right)^{c}} M\left(x, v_{n}(x, 0)\right) \mathrm{d} x \\
& =\int_{\Lambda_{\varepsilon}} f\left(v_{n}(x, 0)\right) v_{n}(x, 0) \mathrm{d} x+o_{n}(1) .
\end{aligned}
$$

Since $\Lambda_{\varepsilon}$ is bounded, by the compactness of sobolev embedding and Lemma 2.3 yield

$$
\lim _{n \rightarrow+\infty} \int_{\Lambda_{\varepsilon}} f\left(v_{n}(x, 0)\right) v_{n}(x, 0) \mathrm{d} x=\int_{\Lambda_{\varepsilon}} f(v(x, 0)) v(x, 0) \mathrm{d} x
$$

and

$$
\lim _{n \rightarrow+\infty} \int_{\Lambda_{\varepsilon}} V(\varepsilon x)\left|v_{n}(x, 0)\right|^{2} \mathrm{~d} x=\int_{\Lambda_{\varepsilon}} V(\varepsilon x)|v(x, 0)|^{2} \mathrm{~d} x
$$

Therefore,

$$
\begin{aligned}
& \limsup _{n \rightarrow+\infty}\left(\int_{\mathbb{R}_{+}^{2}}\left|\nabla v_{n}\right|^{2} \mathrm{~d} x \mathrm{~d} y+\int_{\left(\Lambda_{\varepsilon}\right)^{c}} M\left(x, v_{n}(x, 0)\right) \mathrm{d} x\right) \\
& =\int_{\mathbb{R}_{+}^{2}}|\nabla v|^{2} \mathrm{~d} x \mathrm{~d} y+\int_{\left(\Lambda_{\varepsilon}\right)^{c}} M(x, v(x, 0)) \mathrm{d} x .
\end{aligned}
$$

Now, recalling that $M(x, t) \geq 0$, the Fatous' lemma leads

$$
\begin{aligned}
& \liminf _{n \rightarrow+\infty}\left(\int_{\mathbb{R}_{+}^{2}}\left|\nabla v_{n}\right|^{2} \mathrm{~d} x \mathrm{~d} y+\int_{\left(\Lambda_{\varepsilon}\right)^{c}} M\left(x, v_{n}(x, 0)\right) \mathrm{d} x\right) \\
& \geq \int_{\mathbb{R}_{+}^{2}}|\nabla v|^{2} \mathrm{~d} x \mathrm{~d} y+\int_{\left(\Lambda_{\varepsilon}\right)^{c}} M(x, v(x, 0)) \mathrm{d} x
\end{aligned}
$$

Hence,

$$
\lim _{n \rightarrow+\infty} \int_{\mathbb{R}_{+}^{2}}\left|\nabla v_{n}\right|^{2} \mathrm{~d} x \mathrm{~d} y=\int_{\mathbb{R}_{+}^{2}}|\nabla v|^{2} \mathrm{~d} x \mathrm{~d} y
$$

and

$$
\lim _{n \rightarrow+\infty} \int_{\left(\Lambda_{\varepsilon}\right)^{c}} M(x, v(x, 0)) \mathrm{d} x=\int_{\left(\Lambda_{\varepsilon}\right)^{c}} M(x, v(x, 0)) \mathrm{d} x .
$$

The last limit combined with definition of function $M$ gives

$$
\lim _{n \rightarrow+\infty} \int_{\left(\Lambda_{\varepsilon}\right)^{c}} V(\varepsilon x)\left|v_{n}(x, 0)\right|^{2} \mathrm{~d} x=\int_{\left(\Lambda_{\varepsilon}\right)^{c}} V(\varepsilon x)|v(x, 0)|^{2} \mathrm{~d} x .
$$


Gathering this limit with (3.10), we deduce that

$$
\lim _{n \rightarrow+\infty} \int_{\mathbb{R}} V(\varepsilon x)\left|v_{n}(x, 0)\right|^{2} \mathrm{~d} x=\int_{\mathbb{R}} V(\varepsilon x)|v(x, 0)|^{2} \mathrm{~d} x .
$$

From (3.11)-(3.12),

$$
\lim _{n \rightarrow+\infty}\left\|v_{n}\right\|_{\varepsilon}^{2}=\|v\|_{\varepsilon}^{2}
$$

As $X^{1}\left(\mathbb{R}_{+}^{2}\right)$ is a Hilbert space and $v_{n} \rightarrow v$ weakly in $X^{1}\left(\mathbb{R}_{+}^{2}\right)$, as $n \rightarrow \infty$, the above limit yields

$$
v_{n} \rightarrow v \text { in } X^{1}\left(\mathbb{R}_{+}^{2}\right) \text {, as } n \rightarrow \infty,
$$

showing that $E_{\varepsilon}$ verifies the $(P S)_{c_{\varepsilon}}$.

Theorem 3.6. For $\varepsilon \in\left(0, \varepsilon_{0}\right)$, the functional $E_{\varepsilon}$ has a nonnegative critical point $v_{\varepsilon} \in X^{1}\left(\mathbb{R}_{+}^{2}\right)$ such

$$
E_{\varepsilon}\left(v_{\varepsilon}\right)=c_{\varepsilon} \quad \text { and } \quad E_{\varepsilon}^{\prime}\left(v_{\varepsilon}\right)=0 .
$$

Proof. From Lemma 3.3, there is $\varepsilon_{0}>0$, such that $E_{\varepsilon}$ verifies the $(P S)_{c_{\varepsilon}}$ condition for $\varepsilon \in\left(0, \varepsilon_{0}\right)$. Then, the existence of $v_{\varepsilon}$ is an immediate consequence of the Mountain Pass Theorem due to Ambrosetti and Rabinowitz (see e.g. [28]). The function $v_{\varepsilon}$ is nonnegative, because

$$
E_{\varepsilon}^{\prime}\left(v_{\varepsilon}\right)\left(v_{\varepsilon}^{-}\right)=0 \Longrightarrow v_{\varepsilon}^{-}=0
$$

where $v_{\varepsilon}^{-}=\min \left\{v_{\varepsilon}, 0\right\}$.

Lemma 3.7. Decreasing $\varepsilon_{0}$, if necessary, there are $r, \beta>0$ and $\left(y_{\varepsilon}\right) \subset \mathbb{R}$ such that

$$
\int_{y_{\varepsilon}-r}^{y_{\varepsilon}+r}\left|v_{\varepsilon}(x, 0)\right|^{2} \mathrm{~d} x \geq \beta, \quad \forall \varepsilon \in\left(0, \varepsilon_{0}\right) .
$$

Proof. First of all, we recall that since $\left(v_{\varepsilon}\right)$ satisfies (3.13), there is $\alpha>0$, which is independent of $\varepsilon$, such that

$$
\left\|v_{\varepsilon}\right\|_{\varepsilon}^{2} \geq \alpha, \quad \forall \varepsilon>0
$$

To show (3.14), it is enough to see that for any sequence $\left(\varepsilon_{n}\right) \subset(0,+\infty)$ with $\varepsilon_{n} \rightarrow 0$, the limit below

$$
\lim _{n \rightarrow+\infty} \sup _{y \in \mathbb{R}} \int_{y-r}^{y+r}\left|v_{\varepsilon_{n}}(x, 0)\right|^{2} \mathrm{~d} x=0,
$$

does not hold for any $r>0$. Otherwise, if it holds for some $r>0$, by Lemma 2.4,

$$
\int_{\mathbb{R}} f\left(v_{\varepsilon_{n}}(., 0)\right) v_{n}(x, 0) \mathrm{d} x \rightarrow 0, \text { as } n \rightarrow \infty,
$$

implying that

which contradicts $(3.15)$.

$$
\left\|v_{\varepsilon_{n}}\right\|_{\varepsilon}^{2} \rightarrow 0 \quad \text { as } \quad n \rightarrow+\infty
$$

Lemma 3.8. For any $\varepsilon_{n} \rightarrow 0$, consider the sequence $\left(y_{\varepsilon_{n}}\right) \subset \mathbb{R}$ given in Lemma 3.7 and $\psi_{n}(x, y)=v_{\varepsilon_{n}}\left(x+y_{\varepsilon_{n}}, y\right)$. Then, up to subsequence, there is $\psi \in X^{1}\left(\mathbb{R}_{+}^{2}\right.$ such that

$$
\psi_{n} \rightarrow \psi \quad \text { in } X^{1}\left(\mathbb{R}_{+}^{2}\right) \text {, as } n \rightarrow \infty .
$$

Moreover, there is $x_{0} \in \Lambda$ such that

$$
\lim _{n \rightarrow 0} \varepsilon_{n} y_{\varepsilon_{n}}=x_{0} \quad \text { and } \quad V\left(x_{0}\right)=V_{0} .
$$


Proof. We begin the proof showing that $\left(\varepsilon_{n} y_{\varepsilon_{n}}\right)$ is a bounded sequence. Hereafter, we denote by $\left(y_{n}\right)$ and $\left(v_{n}\right)$ the sequences $\left(y_{\varepsilon_{n}}\right)$ and $\left(v_{\varepsilon_{n}}\right)$ respectively.

Since $E_{\varepsilon_{n}}^{\prime}\left(v_{n}\right) \phi=0, \forall \phi \in X^{1}\left(\mathbb{R}_{+}^{2}\right)$, we have that

$$
\int_{\mathbb{R}_{+}^{2}} \nabla v_{n} \nabla \phi \mathrm{d} x \mathrm{~d} y+\int_{\mathbb{R}} V\left(\varepsilon_{n} x\right) v_{n}(x, 0) \phi(x, 0) \mathrm{d} x-\int_{\mathbb{R}} g_{\varepsilon}\left(x, v_{n}(x, 0)\right) \phi(x, 0) \mathrm{d} x=0 .
$$

Then,

$$
\int_{\mathbb{R}_{+}^{2}}\left|\nabla v_{n}\right|^{2} \mathrm{~d} x \mathrm{~d} y+\int_{\mathbb{R}} V\left(\varepsilon_{n} x\right)\left|v_{n}(x, 0)\right|^{2} \mathrm{~d} x-\int_{\mathbb{R}} g_{\varepsilon}\left(x, v_{n}(x, 0)\right) v_{n}(x, 0) \mathrm{d} x=0 .
$$

From definition of $g$, we see that

$$
g_{\varepsilon}(x, t) \leq f(t), \quad \forall t \geq 0,
$$

and reminding that $v_{n} \geq 0$, we infer that

$$
\int_{\mathbb{R}_{+}^{2}}\left|\nabla v_{n}\right|^{2} \mathrm{~d} x \mathrm{~d} y+\int_{\mathbb{R}} V_{0}\left|v_{n}(x, 0)\right|^{2} \mathrm{~d} x-\int_{\mathbb{R}} f\left(v_{n}(x, 0)\right) v_{n}(x, 0) \mathrm{d} x \leq 0 .
$$

Therefore, there is $s_{n} \in(0,1)$ such that

$$
s_{n} v_{n} \in \mathcal{M}_{0}=\left\{v \in X^{1}\left(\mathbb{R}_{+}^{2}\right) \backslash\{0\}: J_{0}^{\prime}(v) v=0\right\} .
$$

Using the characterization of $c_{0}$, we know that

$$
c_{0} \leq J_{0}\left(s_{n} v_{n}\right), \quad \forall n \in \mathbb{N} .
$$

As

it follows that

$$
J_{0}(w) \leq E_{\varepsilon}(w), \quad \forall w \in X^{1}\left(\mathbb{R}_{+}^{2}\right) \quad \text { and } \quad \varepsilon>0
$$

$$
c_{0} \leq J_{0}\left(s_{n} v_{n}\right) \leq E_{\varepsilon_{n}}\left(s_{n} v_{n}\right) \leq \max _{s \geq 0} E_{\varepsilon_{n}}\left(s v_{n}\right)=E_{\varepsilon_{n}}\left(v_{n}\right)=c_{\varepsilon_{n}} .
$$

Recalling that

$$
c_{\varepsilon_{n}} \rightarrow c_{0}, \text { as } n \rightarrow \infty,
$$

the last inequality gives

$$
\left(s_{n} v_{n}\right) \subset \mathcal{M}_{0}, \forall n \in \mathbb{N}, \quad \text { and } \quad J_{0}\left(s_{n} v_{n}\right) \rightarrow c_{0} \text {, as } n \rightarrow \infty .
$$

By change variable, we also have

$$
\left(s_{n} \psi_{n}\right) \subset \mathcal{M}_{0}, \forall n \in \mathbb{N}, \quad \text { and } \quad J_{0}\left(s_{n} \psi_{n}\right) \rightarrow c_{0}, \text { as } n \rightarrow \infty .
$$

Using Ekeland Variational Principle, we can assume that $\left(s_{n} v_{n}\right)$ is a $(P S)_{c_{0}}$ sequence, that is,

$$
\left(s_{n} \psi_{n}\right) \subset \mathcal{M}_{0}, \forall n \in \mathbb{N}, \quad J_{0}\left(s_{n} \psi_{n}\right) \rightarrow c_{0} \quad \text { and } \quad J_{0}^{\prime}\left(s_{n} \psi_{n}\right) \rightarrow 0, \text { as } n \rightarrow \infty .
$$

A direct computation shows that $\left(s_{n}\right)$ is a bounded sequence with

$$
\liminf _{n \rightarrow+\infty} s_{n}>0 .
$$

Thus, in what follows, we can assume that for some subsequence, there is $s_{0}>0$ such that

$$
s_{n} \rightarrow s_{0}, \text { as } n \rightarrow \infty \text {. }
$$

From definition of $y_{n}$ and $\psi_{n}$, we know that $\psi \in X^{1}\left(\mathbb{R}_{+}^{2}\right) \backslash\{0\}$. Moreover, as $J_{0}^{\prime}\left(s_{n} \psi_{n}\right) \rightarrow 0$, we also have $J_{0}^{\prime}\left(s_{0} \psi\right)=0$. Thereby, by definition of $c_{0}$, we obtain

$$
c_{0} \leq J_{0}\left(s_{0} \psi\right) \text {. }
$$


On the other hand, by Fatou's Lemma we obtain

$$
\liminf _{n \rightarrow+\infty} J_{0}\left(s_{n} \psi_{n}\right) \geq J_{0}\left(s_{0} \psi\right)
$$

which implies

$$
J_{0}\left(s_{0} \psi\right)=c_{0} \quad \text { and } \quad J_{0}^{\prime}\left(s_{0} \psi\right)=0 .
$$

The above equalities combined with Fatous' Lemma, up to a subsequence, gives

$$
s_{n} \psi_{n} \rightarrow s_{0} \psi \quad \text { in } \quad X^{1}\left(\mathbb{R}_{+}^{2}\right) \text {, as } n \rightarrow \infty .
$$

Recalling that $s_{n} \rightarrow s_{0}>0$, as $n \rightarrow \infty$, we can conclude that

$$
\psi_{n} \rightarrow \psi \quad \text { in } X^{1}\left(\mathbb{R}_{+}^{2}\right) \text {, as } n \rightarrow \infty,
$$

showing (3.16).

Using the last limit, we are able to prove (3.17). To do end, we begin making the following claim

Claim 3.1. $\lim _{n \rightarrow+\infty} \operatorname{dist}\left(\varepsilon_{n} y_{n}, \bar{\Lambda}\right)=0$

Indeed, if the claim does not hold, there is $\delta>0$ and a subsequence of $\left(\varepsilon_{n} y_{n}\right)$, still denoted by itself, such that,

$$
\operatorname{dist}\left(\varepsilon_{n} y_{n}, \bar{\Lambda}\right) \geq \delta, \quad \forall n \in \mathbb{N} .
$$

Consequently, there is $r>0$ such that

$$
\left(\varepsilon_{n} y_{n}-r, \varepsilon_{n} y_{n}+r\right) \subset \Lambda^{c}, \quad \forall n \in \mathbb{N} .
$$

From definition of $\psi_{n}$, we have that

$$
\begin{aligned}
& \int_{\mathbb{R}_{+}^{2}}\left|\nabla \psi_{n}\right|^{2} \mathrm{~d} x \mathrm{~d} y+\int_{\mathbb{R}} V\left(\varepsilon_{n} x+\varepsilon_{n} y_{n}\right)\left|\psi_{n}(x, 0)\right|^{2} \mathrm{~d} x \\
& =\int_{\mathbb{R}} g\left(\varepsilon_{n} x+\varepsilon_{n} y_{n}, \psi_{n}(x, 0)\right) \psi_{n}(x, 0) \mathrm{d} x .
\end{aligned}
$$

Note that

$$
\begin{aligned}
& \int_{\mathbb{R}} g\left(\varepsilon_{n} x+\varepsilon_{n} y_{n}, \psi_{n}(x, 0)\right) \psi_{n}(x, 0) \mathrm{d} x \leq \int_{-\frac{r}{\varepsilon_{n}}}^{\frac{r}{\varepsilon_{n}}} g\left(\varepsilon_{n} x+\varepsilon_{n} y_{n}, \psi_{n}(x, 0)\right) \psi_{n}(x, 0) \mathrm{d} x \\
& +\left(\int_{-\infty}^{-\frac{r}{\varepsilon_{n}}}+\int_{\frac{r}{\varepsilon_{n}}}^{+\infty}\right) g\left(\varepsilon_{n} x+\varepsilon_{n} y_{n}, \psi_{n}(x, 0)\right) \psi_{n}(x, 0) \mathrm{d} x
\end{aligned}
$$

and so,

$$
\begin{aligned}
& \int_{\mathbb{R}} g\left(\varepsilon_{n} x+\varepsilon_{n} y_{n}, \psi_{n}(x, 0)\right) \psi_{n}(x, 0) \mathrm{d} x \\
& \leq \frac{V_{0}}{k} \int_{-\frac{r}{\varepsilon_{n}}}^{\frac{r}{\varepsilon_{n}}}\left|\psi_{n}(x, 0)\right|^{2} \mathrm{~d} x+\left(\int_{-\infty}^{-\frac{r}{\varepsilon_{n}}}+\int_{\frac{r}{\varepsilon_{n}}}^{+\infty}\right) f\left(\psi_{n}(x, 0)\right) \psi_{n}(x, 0) \mathrm{d} x .
\end{aligned}
$$

Therefore,

$$
\begin{aligned}
& \int_{\mathbb{R}_{+}^{2}}\left|\nabla \psi_{n}\right|^{2} \mathrm{~d} x \mathrm{~d} y+\int_{\mathbb{R}} V\left(\varepsilon_{n} x+\varepsilon_{n} y_{n}\right)\left|\psi_{n}(x, 0)\right|^{2} \mathrm{~d} x \\
& \leq \frac{V_{0}}{k} \int_{-\frac{r}{\varepsilon_{n}}}^{\frac{r}{\varepsilon_{n}}}\left|\psi_{n}(x, 0)\right|^{2} \mathrm{~d} x+\left(\int_{-\infty}^{-\frac{r}{\varepsilon_{n}}}+\int_{\frac{r}{\varepsilon_{n}}}^{+\infty}\right) f\left(\psi_{n}(x, 0)\right) \psi_{n}(x, 0) \mathrm{d} x .
\end{aligned}
$$


implying that

$$
\int_{\mathbb{R}_{+}^{2}}\left|\nabla \psi_{n}\right|^{2} \mathrm{~d} x \mathrm{~d} y+A \int_{\mathbb{R}^{N}}\left|\psi_{n}(x, 0)\right|^{2} \mathrm{~d} x \leq\left(\int_{-\infty}^{-\frac{r}{\varepsilon_{n}}}+\int_{\frac{r}{\varepsilon_{n}}}^{+\infty}\right) f\left(\psi_{n}(x, 0)\right) \psi_{n}(x, 0) \mathrm{d} x,
$$

where $A=V_{0}\left(1-\frac{1}{k}\right)$. By (3.16),

$$
\left(\int_{-\infty}^{-\frac{r}{\varepsilon_{n}}}+\int_{\frac{r}{\varepsilon_{n}}}^{+\infty}\right) f\left(\psi_{n}(x, 0)\right) \psi_{n}(x, 0) \mathrm{d} x \rightarrow 0, \text { as } n \rightarrow \infty,
$$

and as $n \rightarrow \infty$,

$$
\int_{\mathbb{R}_{+}^{2}}\left|\nabla \psi_{n}\right|^{2} \mathrm{~d} x \mathrm{~d} y+A \int_{\mathbb{R}}\left|\psi_{n}(x, 0)\right|^{2} \mathrm{~d} x \rightarrow \int_{\mathbb{R}_{+}^{2}}|\nabla \psi|^{2} \mathrm{~d} x \mathrm{~d} y+A \int_{\mathbb{R}}|\psi(x, 0)|^{2} \mathrm{~d} x>0,
$$

which contradicts (3.18). This proves Claim 3.1.

From Claim 3.1, there are a subsequence of $\left(\varepsilon_{n} y_{n}\right)$ and $x_{0} \in \bar{\Lambda}$ such that

$$
\lim _{n \rightarrow+\infty} \varepsilon_{n} y_{n}=x_{0}
$$

Claim 3.2. $x_{0} \in \Lambda$.

Indeed, from definition of $\psi_{n}$,

$$
\int_{\mathbb{R}_{+}^{2}}\left|\nabla \psi_{n}\right|^{2} \mathrm{~d} x \mathrm{~d} y+\int_{\mathbb{R}} V\left(\varepsilon_{n} x+\varepsilon_{n} y_{n}\right)\left|\psi_{n}(x, 0)\right|^{2} \mathrm{~d} x \leq \int_{\mathbb{R}} f\left(\psi_{n}(x, 0)\right) \psi_{n}(x, 0) \mathrm{d} x .
$$

Then, by (3.16),

$$
\int_{\mathbb{R}_{+}^{2}}|\nabla \psi|^{2} \mathrm{~d} x \mathrm{~d} y+\int_{\mathbb{R}} V\left(x_{0}\right)|\psi(x, 0)|^{2} \mathrm{~d} x \leq \int_{\mathbb{R}} f(\psi(x, 0)) \psi(x, 0) \mathrm{d} x .
$$

Hence, there is $s_{1} \in(0,1)$ such that

$$
s_{1} \psi \in \mathcal{M}_{V\left(x_{0}\right)}=\left\{v \in X^{1}\left(\mathbb{R}_{+}^{2}\right) \backslash\{0\}: \tilde{J}_{V\left(x_{0}\right)}^{\prime} v=v\right\}
$$

where $\tilde{J}_{V\left(x_{0}\right)}: X^{1}\left(\mathbb{R}_{+}^{2}\right) \rightarrow \mathbb{R}$ is given by

$$
\tilde{J}_{V\left(x_{0}\right)}(v)=\frac{1}{2} \int_{\mathbb{R}_{+}^{2}}|\nabla v|^{2} \mathrm{~d} x \mathrm{~d} y+\frac{1}{2} \int_{\mathbb{R}} V\left(x_{0}\right)|v(x, 0)|^{2} \mathrm{~d} x-\int_{\mathbb{R}} F(v(x, 0)) \mathrm{d} x .
$$

If $\tilde{c}_{V\left(x_{0}\right)}$ denotes the mountain pass level associated with $\tilde{J}_{V\left(x_{0}\right)}$, we must have

$$
\tilde{c}_{V\left(x_{0}\right)} \leq \tilde{J}_{V\left(x_{0}\right)}\left(s_{1} \psi\right) \leq \liminf _{n \rightarrow+\infty} E_{\varepsilon_{n}}\left(v_{n}\right)=\liminf _{n \rightarrow+\infty} c_{\varepsilon_{n}}=c_{0}=\tilde{c}_{V(0)} .
$$

Hence,

from where it follows that

$$
\tilde{c}_{V\left(x_{0}\right)} \leq \tilde{c}_{V(0)}
$$

$$
V\left(x_{0}\right) \leq V(0)
$$

As $V_{0}=\inf _{x \in \mathbb{R}} V(x)$, the above inequality implies that

$$
V\left(x_{0}\right)=V(0)=V_{0} .
$$

Moreover, by $\left(V_{2}\right), x_{0} \notin \partial \Lambda$. Then, $x_{0} \in \Lambda$, finishing the proof. 
Corollary 3.9. Let $\left(\psi_{n}\right)$ the sequence given in Lemma 3.8. Then, $\psi_{n}(\cdot, 0) \in L^{\infty}(\mathbb{R})$ and there is $K>0$ such that

$$
\left|\psi_{n}(\cdot, 0)\right|_{\infty} \leq K, \quad \forall n \in \mathbb{N}
$$

and

$$
\psi_{n}(\cdot, 0) \rightarrow \psi(\cdot, 0) \quad \text { in } \quad L^{p}(\mathbb{R}), \quad \forall p \in(2,+\infty), \text { as } n \rightarrow \infty .
$$

As an immediate consequence, the sequence $h_{n}(x)=g\left(\varepsilon_{n} x+\varepsilon_{n} y_{n}, \psi_{n}(x, 0)\right)$ must verify

$$
h_{n} \rightarrow f(\psi(\cdot, 0)) \quad \text { in } L^{p}(\mathbb{R}), \quad \forall p \in(2,+\infty) \text {, as } n \rightarrow \infty .
$$

Proof. In what follows, for each $L>0$, we set

$$
\psi_{n, L}(x, y)= \begin{cases}\psi_{n}(x, y), & \text { if } \quad \psi_{n}(x, y) \leq L \\ L, & \text { if } \quad \psi_{n}(x, y) \geq L\end{cases}
$$

and

$$
z_{n, L}=\psi_{n, L}^{2(\beta-1)} \psi_{n}
$$

with $\beta>1$ to be determined later. Since

$$
\begin{aligned}
& \int_{\mathbb{R}_{+}^{2}} \nabla \psi_{n} \nabla \phi \mathrm{d} x \mathrm{~d} y+\int_{\mathbb{R}} V\left(\varepsilon_{n} x+\varepsilon_{n} y_{n}\right) \psi_{n}(x, 0) \phi(x, 0) \mathrm{d} x \\
& -\int_{\mathbb{R}} g\left(\varepsilon_{n} x+\varepsilon_{n} y_{n}, \psi_{n}(x, 0)\right) \phi(x, 0) \mathrm{d} x=0, \quad \forall \phi \in X^{1}\left(\mathbb{R}_{+}^{2}\right), \quad \forall n \in \mathbb{N},
\end{aligned}
$$

adapting the same approach explored in C. Alves and G. Figueiredo [3, Lemma 4.1] and using the fact that $\left(\psi_{n}\right)$ is bounded in $X^{1}\left(\mathbb{R}_{+}^{2}\right)$, we conclude that there is $K>0$ such that

$$
\left|\psi_{n}(., 0)\right|_{\infty} \leq K, \quad \forall n \in \mathbb{N} .
$$

Now, the limit (3.20) is obtained by interpolation on the $L^{p}$ spaces, while that (3.21) follows combining the growth condition on $g$ with $(3.20)$.

In what follows, we denote by $\left(w_{n}\right) \subset H^{1 / 2}(\mathbb{R})$ the sequence $\left(\psi_{n}(\cdot, 0)\right)$, that is,

$$
w_{n}(x)=\psi_{n}(x, 0), \quad \forall x \in \mathbb{R} .
$$

Since

$$
\begin{aligned}
& \int_{\mathbb{R}_{+}^{2}} \nabla \psi_{n} \nabla \phi \mathrm{d} x \mathrm{~d} y+\int_{\mathbb{R}} V\left(\varepsilon_{n} x+\varepsilon_{n} y_{n}\right) \psi_{n}(x, 0) \phi(x, 0) \mathrm{d} x \\
& -\int_{\mathbb{R}} g\left(\varepsilon_{n} x+\varepsilon_{n} y_{n}, \psi_{n}(x, 0)\right) \phi(x, 0) \mathrm{d} x=0, \quad \forall \phi \in X^{1}\left(\mathbb{R}_{+}^{2}\right),
\end{aligned}
$$

we have that $w_{n}$ is a solution of the problem

$$
(-\Delta)^{1 / 2} w_{n}+V\left(\varepsilon_{n} x+\varepsilon_{n} y_{n}\right) w_{n}=g\left(\varepsilon_{n} x+\varepsilon_{n} y_{n}, w_{n}\right), \quad \text { in } \quad \mathbb{R},
$$

or equivalently,

$$
(-\Delta)^{1 / 2} u+w_{n}=\chi_{n}, \quad \text { in } \quad \mathbb{R}
$$

where

$$
\chi_{n}(x)=w_{n}(x)+g\left(\varepsilon_{n} x+\varepsilon_{n} y_{n} x, w_{n}(x)\right)-V\left(\varepsilon_{n} x+\varepsilon_{n} y_{n}\right) w_{n}(x), \quad x \in \mathbb{R} .
$$

Denoting $\chi(x)=w(x)+f(w(x))-V\left(x_{0}\right) w(x)$, by Corollary 3.9, we have that

$$
\chi_{n} \rightarrow \chi \quad \text { in } L^{p}(\mathbb{R}), \quad \forall p \in[2,+\infty), \text { as } n \rightarrow \infty,
$$


and there is $k_{1}>0$,

$$
\left|\chi_{n}\right|_{\infty} \leq k_{1}, \quad \forall n \in \mathbb{N} .
$$

Motivated by some results found in [7] ( see also [21] ), which holds for whole line, we deduce that

$$
w_{n}(x)=\left(\mathcal{K} * \chi_{n}\right)(x)=\int_{\mathbb{R}} \mathcal{K}(x-y) \chi_{n}(y) \mathrm{d} y,
$$

where $\mathcal{K}$ is the Bessel kernel, which verifies:

$\left(K_{1}\right) \quad \mathcal{K}$ is positive and even on $\mathbb{R} \backslash\{0\}$,

$\left(K_{2}\right)$ There is $C>0$ such that

$$
\mathcal{K}(x) \leq C /|x|^{2}, \quad \forall x \in \mathbb{R} \backslash\{0\}
$$

and

$\left(K_{3}\right) \quad \mathcal{K} \in L^{q}(\mathbb{R}), \quad \forall q \in L^{q}(\mathbb{R}) \quad \forall q \in[1, \infty]$.

Using the above informations, we are able to prove the following result

Lemma 3.10. The sequence $\left(w_{n}\right)$ verifies

$$
w_{n}(x) \rightarrow 0 \text { as }|x| \rightarrow+\infty,
$$

uniformly in $n \in \mathbb{N}$.

Proof. Given $\delta>0$, we have

$$
\begin{aligned}
0 \leq w_{n}(x) & \leq \int_{\mathbb{R}} \mathcal{K}(x-y)\left|\chi_{n}\right|(y) \mathrm{d} y \\
& =\left(\int_{-\infty}^{x-1 / \delta}+\int_{x+1 / \delta}^{+\infty}\right) \mathcal{K}(x-y)\left|\chi_{n}\right|(y) \mathrm{d} y+\int_{x-1 / \delta}^{x+1 / \delta} \mathcal{K}(x-y)\left|\chi_{n}\right|(y) \mathrm{d} y
\end{aligned}
$$

from $\left(K_{2}\right)$, we have that, for all $n \in \mathbb{N}$,

$$
\begin{aligned}
\left.\int_{-\infty}^{x-1 / \delta}+\int_{x+1 / \delta}^{+\infty}\right) \mathcal{K}(x-y)\left|\chi_{n}\right|(y) \mathrm{d} y & \leq C \delta^{1 / 2}\left|\chi_{n}\right|_{\infty}\left(\int_{-\infty}^{x-1 / \delta}+\int_{x+1 / \delta}^{+\infty}\right) \frac{\mathrm{d} y}{|x-y|^{3 / 2}} \\
& \leq C \delta^{1 / 2} k_{1}\left(\int_{-\infty}^{x-1}+\int_{x+1}^{+\infty}\right) \frac{}{|x-y|^{3 / 2}}=C_{1} \delta^{1 / 2} .
\end{aligned}
$$

On the other hand,

$$
\int_{x-1 / \delta}^{x+1 / \delta} \mathcal{K}(x-y)\left|\chi_{n}\right|(y) \mathrm{d} y \leq \int_{x-1 / \delta}^{x+1 / \delta} \mathcal{K}(x-y)\left|\chi_{n}-\chi\right|(y) \mathrm{d} y+\int_{x-1 / \delta}^{x+1 / \delta} \mathcal{K}(x-y)|\chi|(y) \mathrm{d} y .
$$

Fix $q>1$ with $q$ sufficiently close to 1 and $q^{\prime}>2$ such that $1 / q+1 / q^{\prime}=1$. From $\left(K_{2}\right)$ and $(3.22)$,

$$
\int_{x-1 / \delta}^{x+1 / \delta} \mathcal{K}(x-y)\left|\chi_{n}\right|(y) \mathrm{d} y \leq|\mathcal{K}|_{q}\left|\chi_{n}-\chi\right|_{q^{\prime}}+|\mathcal{K}|_{q}|\chi|_{L^{q^{\prime}}(x-1 / \delta, x+1 / \delta)}
$$

As

$$
\left|\chi_{n}-\chi\right|_{q^{\prime}} \rightarrow 0 \quad \text { as } \quad n \rightarrow+\infty
$$

and

$$
|\chi|_{L^{q^{\prime}}(x-1 / \delta, x+1 / \delta)} \rightarrow 0 \text { as }|x| \rightarrow+\infty,
$$

we deduce that there are $R>0$ and $n_{0} \in \mathbb{N}$ such that

$$
\int_{x-1 / \delta}^{x+1 / \delta} \mathcal{K}(x-y)\left|\chi_{n}\right|(y) \mathrm{d} y \leq \delta, \quad \forall n \geq n_{0} \quad \text { and } \quad|x| \geq R
$$


from (3.26) and (3.27),

$$
\int_{\mathbb{R}} \mathcal{K}(x-y)\left|\chi_{n}\right|(y) \mathrm{d} y \leq C_{1} \delta^{d}+\delta, \quad \forall n \geq n_{0} \quad \text { and } \quad|x| \geq R
$$

The same approach can be used to prove that for each $n \in\left\{1, \ldots, n_{0}-1\right\}$, there is $R_{n}>0$ such that

$$
\int_{\mathbb{R}} \mathcal{K}(x-y)\left|\chi_{n}\right|(y) \mathrm{d} y \leq C_{1} \delta^{d}+\delta, \quad|x| \geq R_{n} .
$$

Hence, increasing $R$, if necessary, we must have

$$
\int_{\mathbb{R}} \mathcal{K}(x-y)\left|\chi_{n}\right|(y) \mathrm{d} y \leq C_{1} \delta^{d}+\delta, \quad \text { for } \quad|x| \geq R, \quad \text { uniformly in } \quad n \in \mathbb{N} .
$$

Since $\delta$ is arbitrary, the proof is finished.

Corollary 3.11. There is $n_{0} \in \mathbb{N}$ such that

$$
v_{n}(x, 0)<a, \quad \forall n \geq n_{0} \quad \text { and } \quad x \in \Lambda_{\varepsilon_{n}}^{c} .
$$

Hence, $u_{n}(x)=v_{n}(x, 0)$ is a solution of $\left(P_{\varepsilon_{n}}^{\prime}\right)$ for $n \geq n_{0}$.

Proof. By Lemma 3.8, we know that $\varepsilon_{n} y_{n} \rightarrow x_{0}$, for some $x_{0} \in \Lambda$. Thereby, there is $r>0$ such that some subsequence, still denoted by itself,

$$
\left(r-\varepsilon_{n} y_{n}, r+\varepsilon_{n} y_{n}\right) \subset \Lambda, \quad \forall n \in \mathbb{N} .
$$

Hence,

$$
\left(y_{n}-r / \varepsilon_{n}, y_{n}+r / \varepsilon_{n}\right) \subset \Lambda_{\varepsilon_{n}}, \quad \forall n \in \mathbb{N},
$$

or equivalently

$$
\Lambda_{\varepsilon_{n}}^{c} \subset\left(-\infty, y_{n}-r / \varepsilon_{n}\right) \cup\left(y_{n}+r / \varepsilon_{n},+\infty\right), \quad \forall n \in \mathbb{N} .
$$

Now, by Lemma 3.10, there is $R>0$ such that

$$
w_{n}(x)<a, \quad \text { for } \quad|x| \geq R \text { and } \forall n \in \mathbb{N},
$$

from where it follows,

$$
v_{n}(x, 0)=\psi_{n}\left(x-y_{n}, 0\right)=w_{n}\left(x-y_{n}\right)<a, \quad \text { for } \quad x \in\left(-\infty, y_{n}-R\right) \cup\left(y_{n}+R,+\infty\right)
$$

and $\forall n \in \mathbb{N}$.

On the other hand, we have that

$$
\Lambda_{\varepsilon_{n}}^{c} \subset\left(-\infty, y_{n}-r / \varepsilon_{n}\right) \cup\left(y_{n}+r / \varepsilon_{n},+\infty\right), \quad \forall n \in \mathbb{N} .
$$

Thus, there is $n_{0} \in \mathbb{N}$, such that

$$
\left(-\infty, y_{n}-r / \varepsilon_{n}\right) \cup\left(y_{n}+r / \varepsilon_{n},+\infty\right) \subset\left(-\infty, y_{n}-R\right) \cup\left(y_{n}+R,+\infty\right), \quad \forall n \geq n_{0},
$$

implying that

$$
v_{n}(x, 0)<a, \quad \forall x \in \Lambda_{\varepsilon_{n}}^{c} \text { and } n \geq n_{0},
$$

finishing the proof. 


\section{Proof of Theorem 1.1}

By Theorem 3.6, we know that problem $(A P)$ has a nonnegative solution $v_{\varepsilon}$ for all $\varepsilon>0$. Applying Corollary 3.11, there is $\varepsilon_{0}$ such that

$$
v_{\varepsilon}(x, 0)<a, \quad \forall x \in \Lambda_{\varepsilon}^{c} \text { and } \forall \varepsilon \in\left(0, \varepsilon_{0}\right),
$$

that is, $v_{\varepsilon}(\cdot, 0)$ is a solution of $\left(P_{\varepsilon}^{\prime}\right)$ for $\varepsilon \in\left(0, \varepsilon_{0}\right)$. Considering

$$
u_{\varepsilon}(x)=v_{\varepsilon}(x / \varepsilon, 0), \quad \text { for } \quad \forall \varepsilon \in\left(0, \varepsilon_{0}\right),
$$

is a solution for original problem $\left(P_{\varepsilon}\right)$.

If $x_{\varepsilon}$ denotes a global maximum point of $u_{\varepsilon}$, it is easy to see that there is $\tau_{0}>0$ such that

$$
u_{\varepsilon}\left(x_{\varepsilon}\right) \geq \tau_{0}, \quad \forall \varepsilon>0 .
$$

In what follows, setting $z_{\varepsilon}=\left(x_{\varepsilon}-\varepsilon y_{\varepsilon}\right) \varepsilon^{-1}$, we have that $z_{\varepsilon}$ is a global maximum point of $w_{\varepsilon}$ and

$$
w_{\varepsilon}\left(z_{\varepsilon}\right) \geq \tau_{0}, \quad \forall \varepsilon>0 .
$$

Now, we claim that

$$
\lim _{\varepsilon \rightarrow 0} V\left(x_{\varepsilon}\right)=V_{0} .
$$

Indeed, by Lemma 3.10, we know that

$$
w_{\varepsilon_{n}}(x) \rightarrow 0 \quad \text { as } \quad|x| \rightarrow+\infty \quad \text { uniformly in } n \in \mathbb{N} .
$$

Therefore, $\left(z_{\varepsilon}\right)$ is a bounded sequence. Moreover, for some subsequence, we also know that there is $x_{0} \in \Lambda$ satisfying $V\left(x_{0}\right)=V_{0}$ and

$$
\varepsilon_{n} y_{\varepsilon_{n}} \rightarrow x_{0} \text {, as } n \rightarrow \infty .
$$

Hence,

implying that

$$
x_{\varepsilon_{n}}=\varepsilon_{n} z_{\varepsilon_{n}}+\varepsilon_{n} y_{\varepsilon_{n}} \rightarrow x_{0}, \text { as } n \rightarrow \infty,
$$

showing that (4.1) holds.

$$
V\left(x_{\varepsilon_{n}}\right) \rightarrow V_{0}, \text { as } n \rightarrow \infty,
$$

\section{REFERENCES}

[1] C.O. Alves, Existence of positive solution for a nonlinear elliptic equation with saddle-like potential and nonlinearity with exponential critical growth in $\mathbb{R}^{2}$ To appear in Milan J. Math.

[2] C. O. Alves, J. M. B. do Ó and O. H. Miyagaki, On nonlinear perturbations of a periodic elliptic problem in $\mathbb{R}^{2}$ involving critical growth, Nonlinear Anal. 56 (2004), 781-791.

[3] C.O. Alves and G.M. Figueiredo, Multiplicity of Positive Solutions for a class of quasilinear problems in $\mathbb{R}^{N}$ via penalization method. Adv. Nonlinear Stud. 5, (2005) 551-572.

[4] C.O. Alves and O. H. Miyagaki, Existence and concentration of solution for a class of fractional elliptic equation in $\mathbb{R}^{N}$ via penalization method, Preprint

[5] A. Ambrosetti and P.H. Rabinowitz, Dual variational methods in critical point theory and applications, J. of Funct. Anal. 14, (1973), 349-381.

[6] B. Barrios, E. Colorado, A. de Pablo, U. Sánchez, On some critical problems for the fractional Laplacian operator, J. Differential Equations 252 (2012), 613-6162.

[7] R.M. Blumenthal and R.K. Getoor, Some theorems on stable processes, Trans. Amer. Math. Soc. 95 (1960), 263-273.

[8] C. Brändle, E. Colorado and U. Sánchez, A concave-convex elliptic problem involving the fractional Laplacian, Proc. R. Soc. Edinb. A. 143 (2013), 39-71.

[9] X. Cabré and Y. Sire, Nonlinear equations for fractional laplacians, I: Regularity,maximum principles, and Hamiltonian estimates, Ann. Inst. H. Poincaré Non Linéare 31 (2014), 23-53.

[10] L. Caffarelli and L. Silvestre, An extension problems related to the fractional Laplacian, Comm. Partial Differential Equation 32 (2007), 1245-1260. 
[11] D.M. Cao, Nontrivial solution of semilinear elliptic equation with critical exponent in $\mathbb{R}^{2}$, Comm. Partial Differential Equation 17 (1992), 407-435.

[12] X. Chang and Z.Q.Wang, Nodal and multiple solutions of nonlinear problems involving the fractional Laplacian, J. Differential Equations 256(2014), 2965-2992.

[13] G. Chen and Y. Zheng, Concentration phenomena for fractional noninear Schrödinger equations, Comm. Pure Appl. Anal. 13 (2014), 2359-2376.

[14] J. Dávila, M. del Pino and J. C. Wei, Concentrating standing waves for the fractional nonlinear Schrödinger equation, J. Differential Equations 256 (2014), 858-892.

[15] D.G. de Figueiredo, O.H. Miyagaki and B. Ruf, Elliptic equations in $\mathbb{R}^{2}$ with nonlinearities in the critical growth range, Calc. Var. Partial Differential Equations 3 (1995), 139-153.

[16] M. del Pino and P.L. Felmer, Local Mountain Pass for semilinear elliptic problems in unbounded domains. Calc. Var. Partial Differential Equations 4 (1996), 121-137.

[17] E. Di Nezza, G. Palatucci and E. Valdinoci, Hitchhiker's guide to the fractional Sobolev spaces, Bull. Sci. Math. 136 (2012), 521-573.

[18] J. M. do Ó, O. H. Miyagaki and M. Squassina, Nonautonomous fractional problems with exponential growth, to appear in NoDEA

[19] J. M. B. do Ó and M.A.S. Souto, On a class of nonlinear Schodinger equations in $\mathbb{R}^{2}$ involving critical growth, J. Differential Equations 174 (2001), 289-311.

[20] M. M. Fall, F. Mahmoudi and E. Valdinoci, Ground states and concentration phenomena for the fractional Schrödinger equation, arXiv:1411.0576v2[math.AP] 24 Apr 2015

[21] P. Felmer, A Quass and J. Tan, Positive solutions of nonlinear Schrödinger equation with the fractional Laplacian, Proc. Roy. Soc. Edinburgh A 142 (2012), 1237-1262.

[22] R. Frank and E. Lenzmann, Uniqueness of non-linear ground states for fractional laplacians in $\mathbb{R}$, Acta Math. 210 (2013), 261-318.

[23] A. Iannizzotto and M. Squassina, 1/2-laplacian problems with exponential nonlinearity, J. Math. Anal. Appl. 414 (2014), 372-385.

[24] T. Ozawa, On critical cases of Sobolev's inequalities, J. Funct. Anal. 127 (1995), 259-269.

[25] S. Secchi, Ground state solutions for nonlinear fractional Schrödinger equations in $\Re^{N}$, J. Math. Phys. 54 (2013), 031501-17 pages.

[26] X. Shang and J. Zhang, Concentrating solutions of nonlinear fractional Schrödinger equation with potentials, J. Differential Equations 258 (2015), 1106-1128.

[27] X. Shang, J. Zhang and Y. Yang, On fractional Schödinger equation in $\Re^{N}$ with critical growth, J. Math. Phys. 54 (2013), 121502-19 pages.

[28] M. Willem,Minimax Theorems, Progress in Nonlinear Differential Equations and their Applications, 24, Birkhäuser 1996.

(C. Alves) Unidade Acadêmica de Matemática, Federal University of Campina Grande, 58429-900, CAmpina Grande-PB, Brazil

E-mail address: coalves@dme.ufcg.edu

(J.M. do Ó) Department of Mathematics, Federal University of Paraíba 58051-900, Jỗo Pessoa-PB, Brazil

E-mail address: jmbo@pq.cnpq.br

(O. Miyagaki) Department of Mathematics, Federal University of Juiz de Fora 36036-330 Juiz de Fora, Minas Gerais, Brazil

E-mail address: olimpio@ufv.br 